\title{
Handwritten Bangla Character Recognition Using Normalized Cross Correlation
}

\author{
Md. Azher Uddin \\ Department of Computer Science and Engineering, International Islamic University Chittagong, Bangladesh.
}

\begin{abstract}
This paper presents a system for recognizing Bangla handwritten characters using template matching algorithm based on normalized cross-correlation. The Bangla alphabets Sorborno and Banjonborno have been chosen to test the recognition System. Character is recognized by analyzing its shape and comparing its features that differentiates each character. The required images are acquired using a digital camera. The color images are then cropped, resized, and converted to binary images. Finally, Template Matching algorithm based on normalized cross-correlation technique is used for recognition of candidate regions. Experimental results show the relatively high accuracy of the developed method. The work has valuable application in the development of an OCR system for handwritten Bangla text.
\end{abstract}

Keywords: Template matching, Normalized Cross-Correlation, OCR, Feature Extraction.

\section{Introduction}

Character recognition is an art of detecting segmenting and identifying characters from image [1]. Optical Character Recognition (OCR) is a process of converting printed or handwritten scanned documents into ASCII characters that a computer can recognize. It contributes immensely to the advancement of automation process and improving the interface between man and machine in many applications. Character recognition is one of the most interesting and fascinating areas of pattern recognition and artificial intelligence [2]. Character recognition is getting more and more attention since last decade due to its wide range of application.

This is an efficient way to turn hard-copy materials into data files that can be edited and otherwise manipulated on a computer. This is the technology used for data entry for business documents, e.g. check clearing, automatic number plate recognition, importing business card information into a contact list, more quickly make textual versions of printed documents, e.g. book scanning for Project Gutenberg, make electronic images of printed documents searchable, e.g. Google Books, converting handwriting in real time to control a computer.

Character recognition process can be categorized in two types. One is Offline character recognition and another one is online character recognition. In offline character recognition system, document is first generated, digitized, stored in computer and then it is processed. While in case of online character recognition system, character is processed while it was under creation. External factors like pressure speed of writing, stroke making etc. does not have any influence in case of offline system but they have great impact on online system.

H. Izakian et. al. [3] used a chain code based algorithm to identify isolated Farsi/Arabic characters. Their method extracts a feature vector of each Farsi characters and computes a chain code for each feature vector using a neighborhood function and a series of normalization. S. A. Husain et al. [4] presented a method for recognition of online Cursive Urdu hand written Nastaliq Script. The system wss currently trained for 250 ligatures. The Recognition rate of base ligatures was 93\%. M Abdul Rahiman et al. [5] has also proposed a Malayalam OCR system. The proposed system has used Daubechies wavelet for feature extraction and neural networks for recognition. The system has been given an accuracy of $92 \%$.

This system can recognize 50 letters with average accuracy of $93.92 \%$. The paper is organized as follows. Section 2 illustrates objective. Section 3 briefly explains Template Matching and normalized crosscorrelation method. Proposed framework is described in section 4. Section 5 presents results Evaluation and Discussion. Finally Conclusion is given in section 6.

\section{Objective}

To presents a simple and novel method for handwritten Bangla character recognition system. Also decrease the recognition problem and the computational time. The goal of Bangla character recognition is to provide an efficient and accurate mechanism to recognize the handwritten Bangla character. My goal is to recognize 50 basic Bangla characters using normalized cross-correlation. 


\section{Template Matching}

Template matching [6] is a method in digital image processing for finding areas of an image that match (are similar) to a template image (patch). It can be used in manufacturing as a part of quality control, a way to navigate a mobile robot, or as a way to detect edges in images.

In template matching we need two primary components:

- $\quad$ Input image (I): The image in which we expect to find a match to the template image and

- $\quad$ Template image $(\mathrm{T})$ : The patch image which will be compared to the template image.

Our goal is to detect the maximum matching area. To identify the matching area, we have to compare the template image against the source image by sliding it. By sliding, we mean moving the patch one pixel at a time (left to right, up to down). At each location, a metric is calculated so it represents how "good" or "bad" the match at that location is.

\subsection{Normalized Cross Correlation}

Normalized cross correlation (NCC) has been commonly used as a metric to evaluate the degree of similarity (or dissimilarity) between two compared images. The main advantage of the normalized cross correlation over the cross correlation is that it is less sensitive to linear changes in the amplitude of illumination in the two compared images. Furthermore, the NCC is confined in the range between -1 and 1 . The setting of detection threshold value is much easier than the cross correlation. It also has applications in pattern recognition, single particle analysis, electron tomographic averaging, cryptanalysis, and neurophysiology.

\section{Proposed Framework}

I have used MATLAB tools to implement the algorithm. The block diagram in figure 1 shows the working approach towards the solution of the stated problem. In this recognition system, I have used normalized cross-correlation technique. For matching the input character with the template image, input candidate character images must be equal sized with the template image. Here the size of template image is $240 \times 160$ pixels.

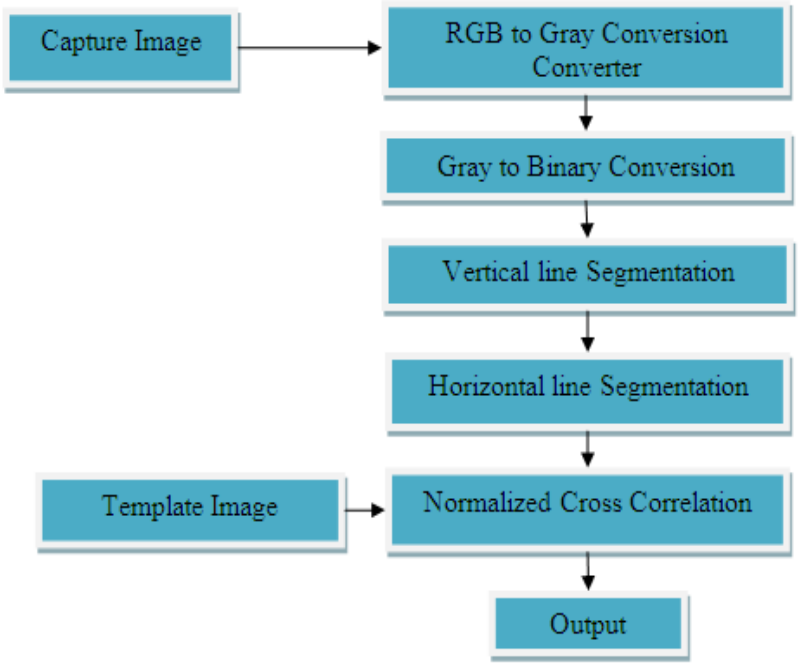

Fig-1: The proposed framework for Handwritten Bangla character recognition.

\subsection{Capture Image}

The handwritten Bangla character is first captured using digital camera and stored in .jpg format. The characters were written by 25 different peoples. The size of the input images is $480 \times 320$ pixels.

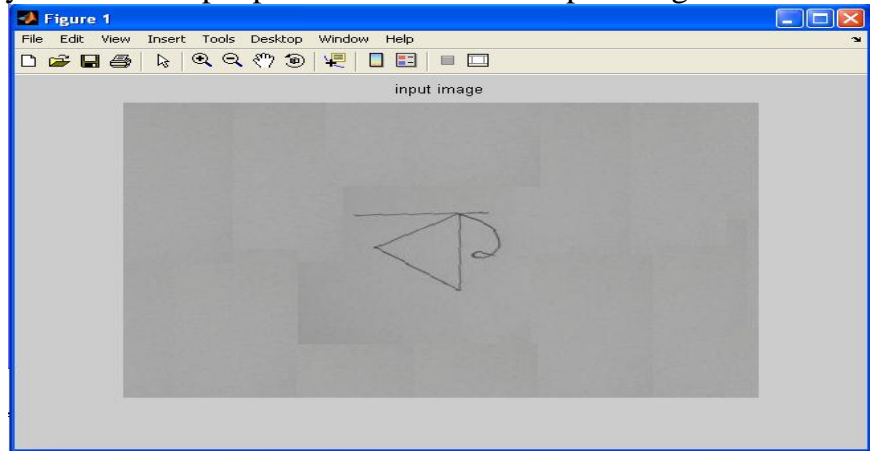

Fig-2: Capture Image 


\subsection{RGB to Gray scale converter}

It is the process that converts the RGB image into Gray scale image. I need to do this conversion because I need binary image for operation. The RGB image is a combination of RED, BLUE AND GREEN colors. The RGB image is 3 dimensional. In an image, at a particular position say (i,j). Image $(\mathrm{i}, \mathrm{j}, 1)$ gives the value of RED pixel, Image( $(\mathrm{i}, \mathrm{j}, 2)$ gives the value of BLUE pixel and Image $(\mathrm{i}, \mathrm{j}, 3)$ gives the value of GREEN pixel. The combination of these primary colors are normalized with $\mathrm{R}+\mathrm{G}+\mathrm{B}=1$; This gives the neutral white color. The grayscale image is obtained from the RGB image by combining 30\% of RED , 60\% of GREEN and $11 \%$ of BLUE. This gives the brightness information of the image. The resulting image will be two dimensional. The value 0 represents black and the value 255 represents white. The range will be between black and white values.

\subsection{Gray scale to Binary image converter}

A binary image is a digital image that has only two possible values for each pixel. Typically the two colors used for a binary image are black and white though any two colors can be used. The color used for the object(s) in the image is the foreground color while the rest of the image is the background color. Thresholding is the simplest method of image segmentation. From a grayscale image, thresholding can be used to create binary images. Thresholding creates binary images from grey-level ones by turning all pixels below some threshold to zero and all pixels about that threshold to one. If $g(x, y)$ is a thresholded version of $f(x, y)$ at some global threshold $T$,

$$
g(x, y)= \begin{cases}1 & \text { if } f(x, y) \leq T \\ 0 & \text { otherwise }\end{cases}
$$

Pixel which have value 1 correspond the object and pixel which have value 0 correspond background. $\mathrm{T}$ is a constants, this approach is called global thresholding.

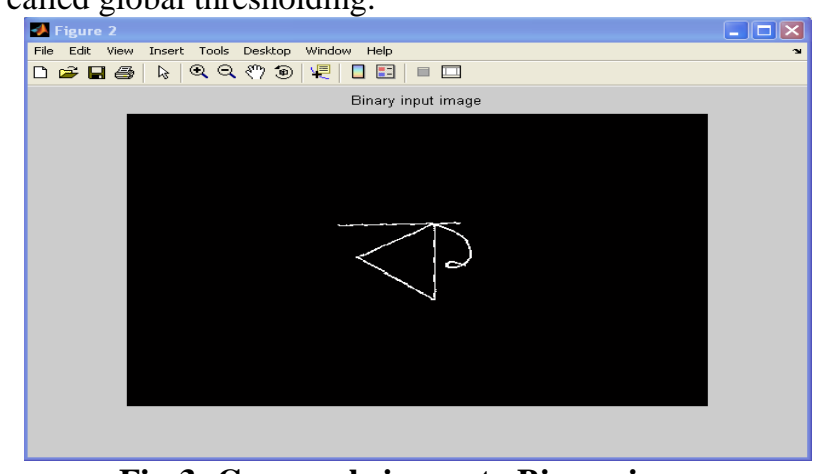

\subsection{Segmentation}

Fig-3: Gray scale image to Binary image

Image segmentation is the process of partitioning a digital image into multiple segments (sets of pixels, also known as superpixels). The goal of segmentation is to simplify and/or change the representation of an image into something that is more meaningful and easier to analyze [7] [8].Image segmentation is typically used to locate objects and boundaries (lines, curves, etc.) in images. More precisely, image segmentation is the process of assigning a label to every pixel in an image such that pixels with the same label share certain visual characteristics.

\subsubsection{Vertical line Segmentation}

In this step to remove non text region first vertical line segmentation is done on the processed binary image. It scans through y-axis of the region.

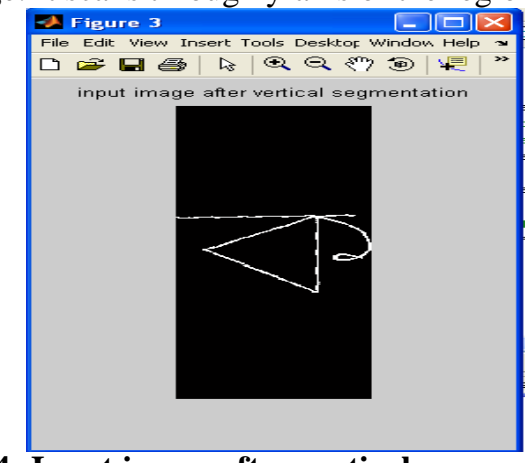

Fig-4: Input image after vertical segmentation

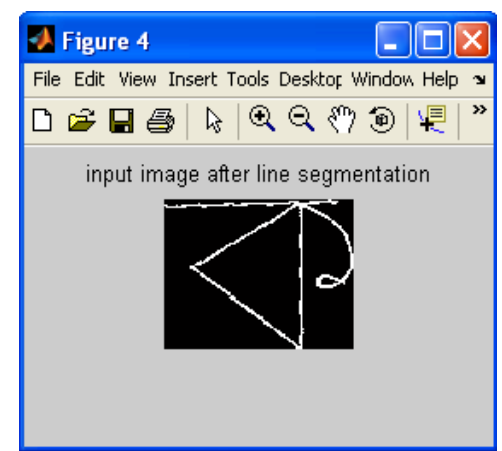

Fig-5: Image after horizontal segmentation 


\subsubsection{Horizontal line Segmentation}

Then horizontal line segmentation is done on the image that is acquired after vertical line segmentation. It scans through $\mathrm{x}$-axis of the region. When scanning discovers any white pixel, it assumes it to be the beginning of any character. And when gets any all-black column in the way of scanning it thinks it to be the end of that digit. Using this handwritten region separated from unwanted area.

\subsection{Normalized Cross Correlation}

To measure the similarity and find the best match, a statistical method correlation is used. Correlation is an effective method for image recognition. This method measures the correlation coefficient between a number of known images with the same sized unknown images or part of an image with the highest correlation coefficient between the images producing the best match.

Let $\mathrm{t}$ be a template image and $\bar{t}$ its average of binary image. Let us assume that $x$ is a candidate handwritten image, having the same size of the template and let $\bar{x}$ be its average of binary image. We use the normalized cross-correlation function between the image pair and define in the discrete case as follows.

$$
N C C=\frac{\sum_{\bar{i}=0}^{m-1} \sum_{j=0}^{n-1}(x-\bar{x})(t-\bar{t})}{\sqrt{\sum_{i=0}^{m-1} \sum_{j=0}^{n-1}(x-\bar{x})^{2} \cdot(t-\bar{t})^{2}}}
$$

Where $N C C$ is the normalized correlation coefficient.

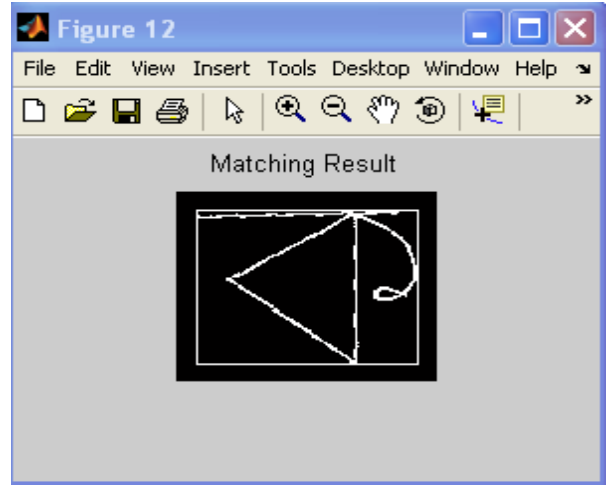

Fig-6: Output result after NCC

\section{Result Evaluation And Discussion}

All experiments were done on dual-core $3.00 \mathrm{GHz}$ with $2 \mathrm{~GB}$ RAM under MATLAB environment. In the experiments, $50 \times 25=1250$ images were employed and the size of the input images is $480 \times 320$ pixels. The Bangla handwritten characters recognition rate is presented in table 1. And the average computational cost for Bangla handwritten characters recognition is shown in Tables 2. Recognition rate is calculated as

\section{Recognition Rate $=\left(\frac{\text { No of recognized letters }}{\text { No of total samples of that letters }}\right) \times 100 \%$}

For example, recognition rate of ' $ক$ ' $=(25 / 25) * 100=100 \%$.

Total recognition rate $=(1174 / 1250) * 100=93.92 \%$.

Figure 2,3,4,5 and 6 shows Illustration of Bangla handwritten characters recognition.

Table 1: Recognition result

\begin{tabular}{|c|c|c|c|}
\hline Letters & $\begin{array}{c}\text { Recognized } \\
\text { Letters }\end{array}$ & $\begin{array}{c}\text { Unrecognized } \\
\text { Letters }\end{array}$ & $\begin{array}{c}\text { Recognition } \\
\text { rate (\%) }\end{array}$ \\
\hline অ & 25 & 0 & $100 \%$ \\
\hline আ & 23 & 2 & $92 \%$ \\
\hline ই & 24 & 1 & $96 \%$ \\
\hline ঈ & 19 & 6 & $76 \%$ \\
\hline ঊ & 24 & 1 & $96 \%$ \\
\hline ঊ & 23 & 2 & $92 \%$ \\
\hline এ & 24 & 1 & $96 \%$ \\
\hline
\end{tabular}




\begin{tabular}{|c|c|c|c|}
\hline ঐ & 24 & 1 & $96 \%$ \\
\hline 3 & 23 & 2 & $92 \%$ \\
\hline ঔ & 23 & 2 & $92 \%$ \\
\hline ঋ & 23 & 2 & $92 \%$ \\
\hline ক & 25 & 0 & $100 \%$ \\
\hline ขr & 25 & 0 & $100 \%$ \\
\hline গ & 24 & 1 & $96 \%$ \\
\hline घ & 23 & 2 & $92 \%$ \\
\hline ঙ & 25 & 0 & $100 \%$ \\
\hline $\bar{\sigma}$ & 25 & 0 & $100 \%$ \\
\hline Б & 22 & 3 & $88 \%$ \\
\hline जा & 22 & 3 & $88 \%$ \\
\hline ঝ & 25 & 0 & $100 \%$ \\
\hline$\Re$ & 25 & 0 & $100 \%$ \\
\hline ট & 25 & 0 & $100 \%$ \\
\hline ঠ & 23 & 2 & $92 \%$ \\
\hline ড & 22 & 3 & $88 \%$ \\
\hline $\bar{\sigma}$ & 25 & 0 & $100 \%$ \\
\hline ศ & 20 & 5 & $80 \%$ \\
\hline $\bar{\nabla}$ & 22 & 3 & $88 \%$ \\
\hline ข & 25 & 0 & $100 \%$ \\
\hline$\overline{7}$ & 25 & 0 & $100 \%$ \\
\hline$\&$ & 23 & 2 & $92 \%$ \\
\hline न & 25 & 0 & $100 \%$ \\
\hline भ & 24 & 1 & $96 \%$ \\
\hline ए & 23 & 2 & $92 \%$ \\
\hline ব & 24 & 1 & $96 \%$ \\
\hline $\bar{\Theta}$ & 21 & 4 & $84 \%$ \\
\hline ম & 22 & 3 & $88 \%$ \\
\hline य & 23 & 2 & $92 \%$ \\
\hline র & 23 & 2 & $92 \%$ \\
\hline ल & 24 & 1 & $96 \%$ \\
\hline ग & 24 & 1 & $96 \%$ \\
\hline य & 23 & 2 & $92 \%$ \\
\hline স & 24 & 1 & $96 \%$ \\
\hline ₹ & 22 & 3 & $88 \%$ \\
\hline ড় & 23 & 2 & $92 \%$ \\
\hline ঢ़ & 23 & 2 & $92 \%$ \\
\hline ग़ & 23 & 2 & $92 \%$ \\
\hline$\rho$ & 25 & 0 & $100 \%$ \\
\hline \% & 22 & 3 & $88 \%$ \\
\hline \% & 25 & 0 & $100 \%$ \\
\hline$\dot{\ddot{b}}$ & 25 & 0 & $100 \%$ \\
\hline
\end{tabular}

Table 2: Average computational cost

\begin{tabular}{|c|c|}
\hline Stage & Average Time (seconds) \\
\hline NCC & $0.336 \mathrm{sec}$ \\
\hline Others & $0.214 \mathrm{sec}$ \\
\hline
\end{tabular}




\begin{tabular}{l|l} 
Total & $0.550 \mathrm{sec}$
\end{tabular}

\section{Conclusion}

In this paper I present a simple model of handwritten Bangla character recognition system using Template Matching algorithm based on Normalized Cross Correlation. The recognition rate of my method is remarkable. The software is implemented by using MATLAB. The input images are taken by a camera and it must be in .jpg format. This system can recognize handwritten Bangla character with an average accuracy of 93.92\%. In the future work, I will try to improve the recognition rate by using Support vector machine and will also try to do handwritten Bangla character recognition from a Bangla text line.

\section{References}

[1]. Mansi Shah \& Gordhan B Jethava “A Literature Review On Hand Written Character Recognition”, Vol -3, Issue -2, March 2013.

[2]. Liu Cheng-Lin, Nakashima, Kazuki, H, Sako, H.Fujisawa, "Handwritten digit recognition: investigation of normalization and feature extraction techniques", Pattern Recognition, Vol. 37, No. 2, pp. 265-279, 2004

[3]. Izakian, H., Monadjemi, S. A., Ladani, B. T. and Zamanifar, K., "Multi-Font Farsi/Arabic Isolated Character Recognition Using Chain Codes," World Academy of Science, Engineering and Technology, 43, 2008.

[4]. S. A. Husain, Asma Sajjad, Fareeha Anwar, "Online Urdu Character Recognition System", MVA 2007 IAPR Conference on Machine Vision Applications, Tokyo, JAPAN, May 16-18, 2007.

[5]. M Abdul Rahiman, M S Rajasree, —OCR for Malayalam Script Using Neural Networks, IEEE, 2009.

[6]. Kai Briechle\&Uwe D. Hanebeck, "Template Matching using Fast Normalized Cross Correlation".

[7]. Linda G. Shapiro and George C. Stockman (2001): "Computer Vision”, pp 279-325, New Jersey, Prentice-Hall, ISBN 0-13030796-3

[8]. Barghout, Lauren, and Lawrence W. Lee. "Perceptual information processing system." Paravue Inc. U.S. Patent Application 10/618,543, filed July 11, 2003.

[9]. Ryo Takei, "A New Grey-Scale Template Image Matching Algorithm Using the Cross-Sectional Histogram Correlation Method", Dynax Corporation Fuchu-City, Fuchu-cho, Tokyo, Japan, 2003.

[10]. Hasan Mohammad Kafi, "Sign Alphabet and Digits Recognition Using Neural network," 2010. 